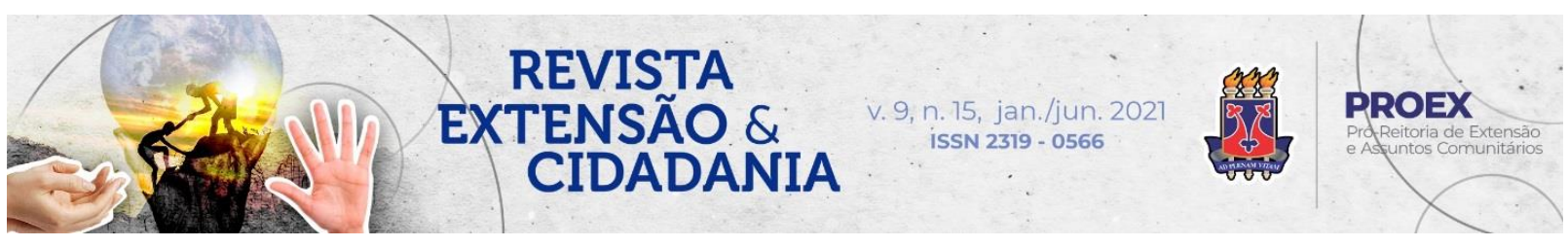

DOI: $10.22481 /$ recuesb.v9i15.8434

\title{
PROJETO DE EXTENSÃO SAÚDE DOS OSSOS - O COMBATE À OSTEOPOROSE: UM RELATO DE EXPERIÊNCIA
}

\section{Extension project "bone health- combating osteoporosis": an experience report}

\author{
Bárbara Assis da Silva ${ }^{1}$ \\ Mateus Elias Sant'Anna Ferreira Ribeiro ${ }^{2}$ \\ Andrey Costa Sartori ${ }^{3}$ \\ Rodrigo de Almeida Ferreira ${ }^{4}$ \\ Eli Ávila Souza Júnior ${ }^{5}$
}

Resumo: Este é um relato de experiência do projeto de extensão "Saúde dos Ossos - o Combate à Osteoporose" que objetivou atuar na promoção da saúde, através de informações sobre a prevenção e tratamento da osteoporose, contribuindo para a transformação social e impactando positivamente na formação discente. As redes sociais foram o instrumento utilizado neste projeto, através das quais, no dia mundial do combate à osteoporose (20 de outubro), cada discente participante publicou uma arte e um texto sobre a doença, orientando a sociedade sobre os riscos dessa doença silenciosa. Aplicou-se um questionário aos discentes participantes após o projeto, sendo que $89,5 \%$ informaram que seus conhecimentos sobre a osteoporose aumentaram e $100 \%$ reconheceram como relevante esse perfil de projeto de extensão. Evidenciou-se, portanto, que o projeto se mostrou relevante no que tange à transformação social, através da disseminação de conteúdo de um tema importante de saúde para a população via mídias sociais, e impactou a formação discente, uma vez que a maioria considerou positiva a adesão ao projeto e tema proposto.

Palavras-chave: Osteoporose. Redes sociais. Educação em saúde.

\footnotetext{
${ }^{1}$ Graduanda em Medicina, Universidade Federal de Alfenas, Alfenas, Minas Gerais, Brasil. Orcid: https://orcid.org/0000-0002-2986 E- mail: baassis2000@hotmail.com

${ }^{2}$ Graduando em Medicina, Universidade Federal de Alfenas, Alfenas, Minas Gerais, Brasil. Orcid: https://orcid.org/0000-0003-2521 E-mail: mateuseliassfr@gmail.com

${ }^{3}$ Graduando em Medicina, Universidade Federal de Alfenas, Alfenas, Minas Gerais, Brasil. Orcid: https://orcid.org/0000-0003-3625-9455 E-mail: andrey.sartori@sou.unifal-mg.edu.br

${ }^{4}$ Médico, Ortopedista e Traumatologista Especialista em Coluna Vertebral. Docente da Universidade Federal de Alfenas, Alfenas, Minas Gerais, Brasil. Orcid: https://orcid.org/0000-0002-2727-0858 E-mail: rodrigortopedia@hotmail.com

${ }^{5}$ Médico, Ortopedista e Traumatologista Especialista em Pé e Tornozelo, Docente, Universidade Federal de Alfenas, Alfenas, Minas Gerais, Brasil. Orcid: https://orcid.org/0000-0002-5054-874X E-mail:

eli.junior@unifal-mg.edu.br
} 
Abstract: This is an experience report of the extension project "Saúde dos Ossos - o Combate à Osteoporose" which aimed to act in the promotion of health, through information on the prevention and treatment of osteoporosis, contributing to social transformation and positively impacting student training. Social networks were the instrument used in this project, through which, on the world day against osteoporosis (October 20), each participating student published an art and a text about the disease, guiding society about the risks of this silent disease. A questionnaire was applied to the participating students after the project, with $89.5 \%$ reported that their knowledge about osteoporosis increased, and $100 \%$ recognized this extension project profile as relevant. It was evident, therefore, that the project proved to be relevant in terms of social transformation, through the dissemination of content on an important health topic for the population via social media, and impacted the student formation, since the majority considered positive the adherence to the project and proposed theme.

Keywords: Osteoporosis. Social media. Health education.

\section{Introdução}

A osteoporose é um notório problema de saúde pública, devido à morbidade e mortalidade associadas às suas complicações, como as fraturas de quadril (fêmur proximal), vértebras, rádio distal, e úmero proximal. Trata-se de uma doença sistêmica, que culmina em redução da massa óssea e danificação da sua microarquitetura, além de incapacidade, fragilidade e maior risco de fraturas.

No contexto epidemiológico, essa doença é mais prevalente nas mulheres brancas após a menopausa, quando comparado a homens e mulheres negras de mesma idade. A taxa de letalidade, no período de um ano, em pacientes com fratura no quadril é de $24 \%$ e, aqueles que sobrevivem, são frequentemente institucionalizados, devido a uma deficiência sustentada (RAY et al., 1997).

A gênese da perda óssea, característica dos quadros de osteoporose, é complexa e multifatorial, sofrendo influência de fatores individuais como a hereditariedade, idade avançada, sexo feminino, baixo peso corporal, etnia, déficit hormonal, etilismo, sedentarismo, tabagismo, e fatores inerentes à nutrição do paciente (CARVALHO; FONSECA; PEDROSA, 2004).

Devido ao alto impacto da doença sobre a funcionalidade e desempenho de atividades diárias, instituiu-se o dia mundial de combate à osteoporose, celebrado no dia 20 de outubro. Segundo Gali (2001), a principal forma de tratamento da osteoporose é a prevenção. Desse modo, foi idealizada uma campanha anual dedicada à conscientização global para prevenção,

Revista Extensão \& Cidadania, v. 9, n. 15, p. 166-174, jan./jun. 2021. 
diagnóstico e tratamento desta doença.

Quando se pensa nos meios de disseminação de informações, Castells (1999) defende que as redes sociais são capazes de se expandir exponencialmente, integrando cada vez mais indivíduos, desde que consigam comunicar-se na rede, ou seja, desde que utilizem os mesmos códigos de comunicação.

O Brasil ocupa a quinta posição no ranking mundial, quando se considera a internet como meio prevalente em que as pessoas mais procuram orientações sobre saúde (VERMELHO et al., 2014). O estudo em questão estima que $81 \%$ das pessoas que possuem acesso à internet, a utilizam como uma maneira de obter informações sobre saúde, remédios e/ou condições que requerem cuidados médicos. Assim, surge um desafio de assegurar que a população brasileira tenha acesso a informações verídicas, de qualidade e comprovadas pela ciência. Desta forma, conclui-se que as ações de promoção da saúde devem ser também divulgadas nas redes sociais digitais.

Assim, considerando-se o amplo acesso de pessoas pertencentes a diversas faixas etárias, às mídias sociais, a busca crescente de informações sobre saúde na internet e a relevância socioeconômica da osteoporose, objetivou-se relatar a experiência do projeto de extensão "Saúde dos Ossos: o Combate à Osteoporose", que atuou na promoção da saúde, através de informações sobre a prevenção e tratamento da osteoporose, contribuindo para a transformação social, assim como no impacto à formação discente.

\section{Métodos}

Trata-se de um relato de experiência do projeto de extensão "Saúde dos Ossos: o combate à osteoporose", pertencente à Faculdade de Medicina de uma universidade pública de Minas Gerais, devidamente registrado na Pró-reitoria de Extensão da Universidade sob o protocolo PREAE 4686.

Participaram do projeto de extensão 101 estudantes de Medicina de todos os anos da Faculdade de Medicina. As atividades de extensão foram realizadas no Dia Mundial de Combate à Osteoporose (20 de outubro de 2020). Os alunos de Medicina interessados se inscreveram no projeto através do CAEX (Controle de Ações de Extensão) da universidade.

As ferramentas utilizadas foram as redes sociais Instagram e Facebook. Aqueles que se inscreveram no projeto postaram em suas mídias sociais (Instagram e Facebook) a arte e texto produzidos pelos idealizadores do projeto, de modo a divulgar, para a comunidade um pouco

Revista Extensão \& Cidadania, v. 9, n. 15, p. 166-174, jan./jun. 2021. 
sobre essa doença. A seguir, o texto de divulgação:

\section{"20 de outubro - DIA INTERNACIONAL DO COMBATE A OSTEOPOROSE"}

A osteoporose é uma doença osteometabólica caracterizada pela perda progressiva de densidade óssea, gerando fragilidade dos ossos, tornando o indivíduo vulnerável a fraturas. Estudos realizados pelo Ministério da Saúde e pela Fundação Internacional de Osteoporose (IOF) revelaram que a doença atinge cerca de 10 milhões de brasileiros atualmente. As mulheres são as mais acometidas, principalmente na faixa etária acima de 50 anos. Ademais, demonstrou-se que $75 \%$ dos pacientes portadores da osteoporose só são diagnosticados após a primeira fratura. POR ISSO, É PRECISO SE PREVENIR! A recomendação é que a prevenção se inicie antes dos 40 anos, principalmente nas mulheres. É preciso consumir alimentos que forneçam as quantidades ideais de cálcio, vitamina $\mathrm{D}$ e outros elementos como magnésio e fósforo. Tais componentes podem ser encontrados no dia a dia em: leite e derivados, verduras verdes, leguminosas, peixes, fígado e cogumelos. Além disso, a prática regular de exercícios físicos é essencial, destacando-se os exercícios de impacto como caminhadas e corridas, uma vez que estimulam a deposição óssea e o fortalecimento muscular. Destaca-se, ainda, a exposição segura diária ao sol como fonte natural de produção de vitamina $\mathrm{D}$, e o abandono de práticas não saudáveis como o tabagismo e o alcoolismo. O rastreamento da osteoporose, baseado em exames complementares como a densitometria óssea e exames laboratoriais, deve ser realizado em grupos de risco a fim de detectar a doença inicialmente, permitir o seu tratamento precoce, e prevenir os desfechos negativos como fraturas por insuficiência. Indague ao seu médico se você faz parte desse grupo! Cuide da sua saúde óssea! A prevenção é o melhor remédio! Informe-se e previna-se; a osteoporose é uma doença silenciosa e que pode causar sérias consequências. Esse texto foi produzido no Projeto de Extensão SAÚDE DOS OSSOS: O COMBATE À OSTEOPOROSE da UNIFAL-MG."’

A figura 1 ilustra a imagem divulgada nas redes sociais dos alunos participantes.

Revista Extensão \& Cidadania, v. 9, n. 15, p. 166-174, jan./jun. 2021. 


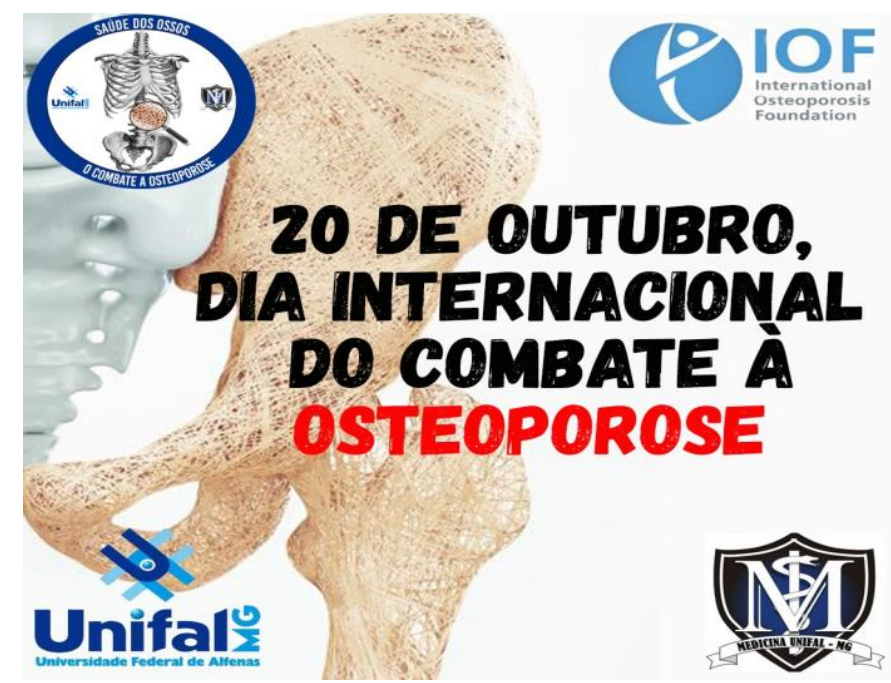

Fonte: Elaboração dos autores.

Após a execução, um questionário foi elaborado pelos professores coordenadores e aplicado aos participantes, com o objetivo de avaliar o projeto de extensão. As perguntas envolveram: conhecimento prévio sobre a existência do dia mundial de combate à osteoporose, relevância das campanhas de conscientização online, ganho de conhecimento sobre o tema e recomendação deste tipo de ação para outras áreas. Foram obtidas 59 respostas.

\section{Resultados}

Dos 59 participantes que responderam ao questionário, apenas 1 tinha conhecimento sobre a existência de um dia específico do ano (20 de outubro) para campanhas de combate à osteoporose. Além disso, 100\% responderam que atualmente consideram que as campanhas online são importantes para a conscientização da população, tais como a desenvolvida pelo referido projeto.

Todos os estudantes indagados recomendam esse tipo de campanha online de conscientização para outras áreas médicas e patologias, além da ortopedia e osteoporose. As demais perguntas do questionário estão descritas no quadro a seguir.

Revista Extensão \& Cidadania, v. 9, n. 15, p. 166-174, jan./jun. 2021. 
Quadro 1 - Conhecimento dos alunos sobre osteoporose, Alfenas, Minas Gerais,

Brasil, 2020. $(\mathbf{N}=59)$

\begin{tabular}{|l|l|l|}
\hline \multicolumn{1}{|c|}{ Perguntas } & \multicolumn{1}{c|}{ Sim } & \multicolumn{1}{c|}{ Não } \\
\hline Seus conhecimentos sobre a osteoporose e sua prevenção aumentaram? & $89,5 \%$ & $10,5 \%$ \\
\hline $\begin{array}{l}\text { Você reconhece a osteoporose como um problema de saúde pública } \\
\text { relevante? }\end{array}$ & $100 \%$ & $0 \%$ \\
\hline Você sabia que a osteoporose é uma doença silenciosa? & $89,5 \%$ & $10,5 \%$ \\
\hline $\begin{array}{l}\text { Você sabia que a maioria dos pacientes acometidos só são diagnosticados } \\
\text { após a primeira fratura? }\end{array}$ & $47,4 \%$ & $52,6 \%$ \\
\hline
\end{tabular}

Fonte: Elaborado pelos autores.

Por fim, foi pedido que os alunos deixassem comentários sobre a relevância do projeto, incluindo críticas, elogios e sugestões. O feedback, em geral, foi positivo, entretanto, sugeriuse que o texto de divulgação não fosse tão extenso.

\section{Discussão}

Para Sousa, Miota e Carvalho (2011), é necessário que o docente utilize das grandes quantidades de recursos tecnológicos digitais, tais quais as redes sociais, para a propagação de informação que possa ser utilizada em sua prática pedagógica. Nesse cenário, insere-se o projeto "Saúde dos Ossos - o Combate à Osteoporose", em que os materiais foram construídos com intuito de propagar o saber à sociedade, assim como otimizar o aprendizado discente, a partir das tecnologias digitais.

De acordo com os resultados obtidos, a maioria dos acadêmicos aprovou esse modelo de campanha de conscientização online, considerando-o passível de ser aplicado em outras áreas médicas e patológicas. Os dados mostram ainda que, antes do projeto, os conhecimentos sobre a temática eram defasados, o que corroborou para o grande aprendizado alcançado pelos participantes.

A educação em saúde, para Oliveira e Gonçalves (2004), é uma importante dimensão da prevenção, representada por ações que se preocupam com a melhoria das condições de vida e saúde das populações as quais atendem. As iniciativas de educação em saúde, como o projeto de extensão "Saúde dos Ossos: o Combate à Osteoporose", contribuem para que as pessoas

Revista Extensão \& Cidadania, v. 9, n. 15, p. 166-174, jan./jun. 2021. 
desenvolvam a autossuficiência para identificar problemas inerentes a sua saúde e utilizar meios para melhorar e preservar a sua qualidade de vida. Dessa maneira, a aquisição de informações sobre os determinantes de saúde e doença desencadeiam um processo em que os indivíduos (agora sujeitos, graças à conscientização), buscam a sua autonomia para o enfrentamento dos seus problemas em direção a uma vida mais saudável. Esse processo pode ser visto como um empoderamento.

Oliveira e Gonçalves (2004) consideram que a educação em saúde pode ser realizada tanto através de aconselhamentos interpessoais, quanto daqueles impessoais, sendo estes últimos os que acontecem através da mídia, com objetivo de atingir o maior número possível de pessoas. Essa foi a idealização do projeto em questão: levar conhecimento, já que a educação está atrelada à aprendizagem, provocando, assim mudanças de pensamentos e atitudes. Ademais, buscou-se a comunicação com a população de acordo com a sua realidade, e, por tal motivo, utilizaram-se as redes sociais mais populares no Brasil em linguagem simples nas postagens.

Para além da importância desta ação no que tange a educação, é imprescindível também conhecer os tópicos mais relevantes sobre a osteoporose, visto que é um quadro possível de ser prevenido: através de alimentação rica em cálcio, vitamina $\mathrm{D}$, fósforo e potássio; prática regular de exercícios físicos; cessação do alcoolismo e tabagismo. Além disso, é importante que pessoas de diferentes faixas etárias tenham acesso a tais informações, uma vez que os cuidados devem começar logo na infância, pois é nesta fase que a formação de massa óssea está em seu ápice (COMPSTON; McCLUNG; LESLIE, 2019). É importante, também, enfatizar a disseminação desse tema entre as mulheres, uma vez que são parte do gênero mais acometido (CARVALHO; FONSECA; PEDROSA, 2004).

Do ponto de vista econômico, a prevenção é, também, de suma importância para os cofres públicos, uma vez que os gastos em saúde, associados às fraturas que decorrem da osteoporose, somam cerca de 60 bilhões de dólares, por ano, apenas nos Estados Unidos (SEDLAK et al., 2000). Tal estudo vai ao encontro dos dados encontrados pelos estudos de Ray (1997), que demonstraram que somente as despesas médicas ligadas aos serviços de internação representam mais de $90 \%$ de todos os gastos decorrentes das fraturas osteoporóticas.

A educação em saúde é essencial para a reflexão e mudança de comportamento na vida dos indivíduos. Assim, a mesma precisa ser sistematicamente planejada e assumida como um papel importante na atuação do profissional médico. Por fim, diante do cenário mundial

Revista Extensão \& Cidadania, v. 9, n. 15, p. 166-174, jan./jun. 2021. 
marcado pela pandemia de SARS-CoV-2 (MAIA, DIAS, 2020), é inegável o papel de destaque que as redes sociais ganham como ferramentas para a disseminação de conhecimento. O projeto de extensão "Saúde dos Ossos: o Combate à osteoporose" não é pioneiro na divulgação de informações sobre saúde nas mídias digitais, mas corroborou na nova tendência mundial de ensino e aprendizagem.

\section{Conclusão}

Evidenciou-se que o projeto de extensão "Saúde dos Ossos - o Combate à Osteoporose" mostrou-se relevante no que tange à transformação social, através da disseminação de conteúdo relevante via mídias sociais, e impactou a formação discente, uma vez que a maioria considerou positiva a adesão ao projeto e tema proposto.

\section{Referências}

CARVALHO, C. M. R. G. de; FONSECA, C. C. C.; PEDROSA, J. I. Educação para a saúde em osteoporose com idosos de um programa universitário: repercussões. Caderno Saúde Pública, Rio de Janeiro, v. 20, n. 3, p. 719-726, 2004.

CASTELLS, M. A sociedade em rede. A Era da Informática: Economia, Sociedade e Cultura. Rio de Janeiro: Paz e Vida, 1999.

COMPSTON, J. E.; McCLUNG, M. R.; LESLIE, W. D. Osteoporosis. The Lancet, v. 393, p. 364-376, 2019.

GALI, J. C. et al. Osteoporose. Acta Ortopédica Brasileira, v. 9, n. 2, p. 53-62, 2001.

INTERNATIONAL OSTEOPOROSIS FOUNDATION. Disponível em: https://www.osteoporosis.foundation/. Acesso em: 5 abr. 2021.

MAIA, B. R.; DIAS, P. C. Ansiedade, depressão e estresse em estudantes universitários: o impacto da COVID-19. Estudos de Psicologia, Campinas, v. 37, 2020.

OLIVEIRA, H. M. de; GONÇALVES, M. J. F. Educação em saúde: uma experiência transformadora. Revista Brasileira de Enfermagem, Brasília, v. 57, n. 6, p. 761-763, 2004.

RAY, N. F. et al. Medical Expenditures for the Treatment of Osteoporotic Fractures in the United States in 1995: Report from the National Osteoporosis Foundation. Journal of Bone and Mineral Research, v. 12, n. 1, p. 24-35, 1997.

ROLNICK, S. J. et al. What is the impact of osteoporosis education and bone mineral density

Revista Extensão \& Cidadania, v. 9, n. 15, p. 166-174, jan./jun. 2021. 
testing for postmenopausal women in a managed care setting? Menopause: The Journal of The North American Menopause Society, v. 8, n. 2, p. 141-148, 2001.

SEDLAK, C. A. et al. Osteoporosis Education Programs: Changing Knowledge and Behaviors. Public Health Nursing, v.17, n. 5, p. 398-402, 2000.

SOUSA, R.; MIOTA, F. M. C. das S. C.; CARVALHO, A. B. G. (org.). Tecnologias digitais na educação. Campina Grande: EDUEPB, 2011.

VERMELHO, S. C. et al. Refletindo sobre as redes sociais digitais. Educação \& Sociedade, Campinas, v. 35, n. 126, p. 179-196, 2014.

Recebido: 12.042021

Aceito: 19.06.2021

Revista Extensão \& Cidadania, v. 9, n. 15, p. 166-174, jan./jun. 2021. 